\title{
Seismology of Procyon A: determination of mode frequencies, amplitudes, lifetimes, and granulation noise
}

\author{
S. Leccia ${ }^{1}$, H. Kjeldsen ${ }^{2}$, A. Bonanno ${ }^{3}$, R. U. Claudi ${ }^{4}$, R. Ventura ${ }^{3}$, and L. Paterno ${ }^{5}$ \\ 1 INAF - Astronomical Observatory of Capodimonte, Salita Moiariello 16, 80131 Napoli, Italy \\ e-mail: leccia@na.astro.it \\ 2 Department of Physics and Astronomy, University of Aarhus, Ny Munkegade, Building 1520, 8000 Aarhus C, Denmark \\ 3 INAF - Astrophysical Observatory of Catania, via S. Sofia 78, 95123 Catania, Italy \\ 4 INAF - Astronomical Observatory of Padua, Vicolo Osservatorio 5, 35122 Padova, Italy \\ 5 Department of Physics and Astronomy, Astrophysics Section, University of Catania, via S. Sofia 78, 95123 Catania, Italy
}

Received 8 September 2006 / Accepted 7 December 2006

\section{ABSTRACT}

The F5 IV-V star Procyon A ( $\alpha-\mathrm{CMi})$ was observed in January 2001 by means of the high-resolution spectrograph SARG operating with the TNG $3.5 \mathrm{~m}$ Italian telescope (Telescopio Nazionale Galileo) in the Canary Islands, exploiting the iodine cell technique. The time series of about 950 spectra carried out during 6 observation nights and a preliminary data analysis (Claudi et al. 2005) showed a significant power excess between 0.5 and $1.5 \mathrm{mHz}$, with $\simeq 1 \mathrm{~m} \mathrm{~s}^{-1}$ peak amplitude. Here we present a more detailed analysis of the time series, based on both radial velocity and line equivalent width analyses. From the power spectrum we found a typical $p$-mode frequency comb-like structure, identified 11 frequencies with a good margin of certainty in the interval 500-1400 $\mu \mathrm{Hz}$ of modes with $l=0,1,2$ and $7 \leq n \leq 22$, and determined large and small frequency separations, $\Delta v=55.90 \pm 0.08 \mu \mathrm{Hz}$ and $\delta v_{02}=7.1 \pm 1.3 \mu \mathrm{Hz}$, respectively. The mean amplitude per mode $(l=0,1)$ at peak power is $0.45 \pm 0.07 \mathrm{~m} \mathrm{~s}^{-1}$, twice larger than the solar one, and the mode lifetime is $2 \pm 0.4 \mathrm{~d}$, which indicates a non-coherent, stochastic source of mode excitation. Line equivalent width measurements do not show a significant excess in power in the examined spectral region but allowed us to infer an upper limit to the granulation noise.

Key words. stars: oscillations - stars: individual: Procyon A - techniques: spectroscopic - techniques: radial velocities

\section{Introduction}

Procyon A ( $\alpha$-CMi, HR 2943, HD61421), hereinafter simply called Procyon, is a F5 IV-V star with $V=0.363$ at a distance of $3.53 \mathrm{pc}$ in a $40 y$-period visual binary system, the companion being a white dwarf more than 10 mag fainter. By adopting the very accurate parallax measured by HIPPARCOS, $\Pi=285.93 \pm 0.88$ mas, Allende Prieto et al. (2002) derived a mass of $1.42 \pm 0.06 M_{\odot}$, a radius of $2.071 \pm 0.02 R_{\odot}$, and a gravity $\log g=3.96 \pm 0.02$.

Owing to its proximity and brightness, Procyon has already attracted the attention of stellar seismologists (Brown et al. 1991; Guenther \& Demarque 1993; Barban et al. 1999; Chaboyer et al. 1999; Martić et al. 1999, 2004; Eggenberger et al. 2004), causing an intense debate among scientists. The power excess in the range $0.5-1.5 \mathrm{mHz}$ found by Bouchy et al. (2004), Martić et al. (2004), Eggenberger et al. (2004), and Claudi et al. (2005, hereinafter referred to as Paper I) seems to have a stellar origin and is consistent with a $p$-mode comb-like pattern.

However, data from the Canadian MOST satellite (Matthews et al. 2004) show no significant power excess in the same spectral region. In this regard, Bedding et al. (2005) suggest that the most likely explanation for the null detection could be a dominating non-stellar noise source in the MOST data, although Régulo \& Roca Cortés (2005) make a claim for the presence of a signal in these data.

In Paper I we presented high precision radial velocity (RV) measurements carried out during 6 observation nights by means of the high resolution spectrograph SARG operating with the TNG 3.5 m Italian telescope (Telescopio Nazionale Galileo) in the Canary Islands. The data showed a power excess between $0.5-1.5 \mathrm{mHz}$ with a large separation of about $56 \pm 2 \mu \mathrm{Hz}$, in agreement with previous measurements by Mosser et al. (1998), Barban et al. (1999), Martić et al. (1999, 2004), and Eggenberger et al. (2004), who found values in the range $53-56 \mu \mathrm{Hz}$. Here we deal with an improvement in the preliminary analysis presented in Paper I concerning an accurate time-series analysis of both radial velocities and line equivalent width $(E W)$ measurements, from which mode frequencies, amplitudes, and lifetimes are determined and constraints on stellar granulation noise are deduced.

\section{Observations}

SARG is a high-resolution cross-dispersed echelle spectrograph (Gratton et al. 2001) that operates in both single-object and longslit (up to $26^{\prime \prime}$ ) observing modes and covers a spectral wavelength range from $370 \mathrm{~nm}$ up to about $1000 \mathrm{~nm}$, with a resolution ranging from $R=29000$ up to $R=164000$. Our spectra were obtained at $R=144000$ in the wavelength range between $462-792 \mathrm{~nm}$. The calibration iodine cell works only in the blue part of the spectrum $(462-620 \mathrm{~nm})$ that has been used for measuring Doppler shifts. The red part of the echelle spectrum of Procyon was used for measuring $E W$ of absorption lines sensitive to temperature. During the observing period we collected about 950 high signal-to-noise ratio $(S / N)$ spectra with a mean 
exposure time of about $10 \mathrm{~s}$ (see Paper I for more details). The red part spectrum was calibrated in wavelength by using a Th-Ar lamp. The analysis of both the blue and red parts of the spectrum was performed by using the IRAF package facilities.

\section{Radial velocity measurements and analysis}

RV have already been determined by means of the AUSTRAL code (Endl et al. 2000) which models instrumental profile, stellar, and iodine-cell spectra in order to measure Doppler shifts (see also Paper I). This code also provides an estimate of the uncertainty in the velocity measurements, $\sigma_{i}$. These values were derived from the scatter of velocities measured from many $(\simeq 100)$ small $(\simeq 2 \AA)$ segments of the echelle spectrum. To perform a weighted Fourier analysis of the data, we first verified that these $\sigma_{i}$ values reflected the noise properties of the velocity measurements, following the Butler et al. (2004) approach. The high-frequency noise in the power spectrum (PS), well beyond the stellar signal, reflects the properties of the noise in the data; since we do expect the oscillation signal the dominant cause of variations in the velocity time series, we need to remove it before analyzing the noise. We do that iteratively by finding the highest peak in the PS of the velocity time-series and subtracting the corresponding sinusoid from the time-series. This procedure is carried out for the strongest peaks in the oscillation spectrum in the frequency range $0-2 \mathrm{mHz}$, until the spectral leakage into high frequencies from the remaning power is negligible. Thus we have a time series of residual velocities, $r_{i}$, that reflects the noise properties of the measurements. We then analyze the ratio $r_{i} / \sigma_{i}$, which is expected to be Gaussiandistributed, so that the outliers correspond to the suspected data points. The cumulative histogram of $\left|r_{i} / \sigma_{i}\right|$ is shown in the upper panel of Fig. 1, along with the cumulative histogram for the best-fit Gaussian distribution. A significant excess of outliers is evident for $\left|r_{i} / \sigma_{i}\right| \geq 2$. The lower panel of Fig. 1 shows the ratio of the values of the observed points to the corresponding ones of the Gaussian curve, i.e. the fraction $f$ of data points that could be considered as "good" observations, namely those close to the unity. The quantities $w_{i}=1 /\left(\sigma_{i}^{2} f\right)$ were adopted as weights in the computation of the weighted PS shown in Fig. 2, where the most prominent peak has an amplitude of $\simeq 1 \mathrm{~m} \mathrm{~s}^{-1}$.

\subsection{Search for a comb-like pattern}

In solar-like stars, $p$-mode oscillations of low harmonic degree, $l$, and high radial order, $n$, are expected to produce a characteristic comb-like structure in the PS with mode frequencies $v_{n, l}$ that are reasonably well approximated by the Tassoul's simplified asymptotic relationship (Tassoul 1980):

$v_{n, l} \simeq \Delta v(n+l / 2+\varepsilon)-l(l+1) \delta v_{02} / 6$

where $\Delta v=\left\langle v_{n, l}-v_{n-1, l}\right\rangle$ and $\delta v_{02}=\left\langle v_{n, 0}-v_{n-1,2}\right\rangle$ are the average large frequency separation and small frequency separation for $l=0,2$, respectively, and $\varepsilon$ is a constant of the order of unity, sensitive to the sound speed near the surface layers of the star. The value of $\Delta v$ reflects the average stellar density, while $\delta v_{02}$ is largely determined by the conditions in the core of the star and reflects its evolutionary state. In order to estimate the large frequency separation $\Delta v$ in the region of the power excess detected in the PS, we applied the comb-response method, which is a generalization of the PS of a PS and consequently allows us to search for regularity in any spectral pattern. The comb-response
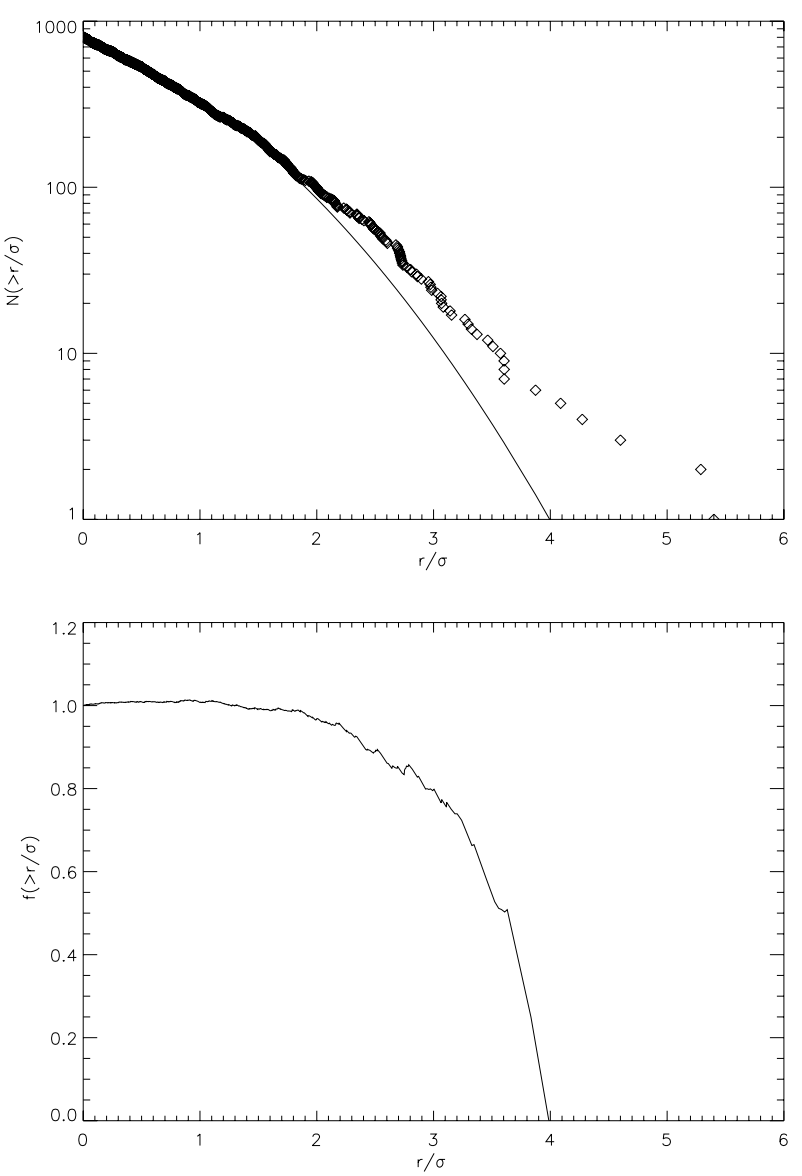

Fig. 1. Upper panel: cumulative histograms of $\left|r_{i} / \sigma_{i}\right|$ for SARG data. The diamonds show the observed data, and the solid curve shows the result expected for a Gaussian-distributed noise. Lower panel: ratio of the observed to the expected histograms, indicating the fraction of "good" data points.

function, $\mathrm{CR}(\Delta v)$, is defined by Kjeldsen et al. (1995) as the following products of PS calculated for a series of trial values $\Delta v$ :

$$
\begin{aligned}
\mathrm{CR}(\Delta v)= & \operatorname{PS}\left(\xi_{-1 / 2}\right) \operatorname{PS}\left(\xi_{+1 / 2}\right) \operatorname{PS}\left(\xi_{-1}\right) \operatorname{PS}\left(\xi_{+1}\right) \\
& \times\left[\operatorname{PS}\left(\xi_{-3 / 2}\right) \operatorname{PS}\left(\xi_{+3 / 2}\right) \operatorname{PS}\left(\xi_{-2}\right) \operatorname{PS}\left(\xi_{+2}\right)\right]^{1 / 2}
\end{aligned}
$$

where $\xi_{ \pm k}=v_{\max } \pm k \Delta v$, with $v_{\max }$ the frequency of the highest peak in the PS. A peak in the CR at a particular spacing $\Delta v$ indicates the presence of a regular series of peaks in the PS, centered at the central frequency $v_{\max }$ - tentatively assumed to be an oscillation mode - and having a spacing $\Delta v / 2$. In order to select the central frequencies $v$ for the CR analysis, we evaluated the white noise in the PS between $2.5-3.0 \mathrm{mHz}$ and adopted as $v_{\max }$ the frequencies of the peaks in the PS between $0.5-1.5 \mathrm{mHz}$ with $S / N>4$. For each central frequency $v_{\max }$, we searched for the maximum $\mathrm{CR}$ in the range $20 \leq \Delta v \leq 80 \mu \mathrm{Hz}$. Figure 3 reports the $\Delta v$ 's determined from the $\mathrm{CR}$ analysis, which gives a mean value $\Delta v=55.7 \pm 1.4 \mu \mathrm{Hz}$, where the error is the standard deviation, and Fig. 4 depicts the cumulative CR function computed as the sum of all the individual $\mathrm{CR}$ functions referring to each central frequency $v_{\max }$, which gives $\Delta v=56 \pm 1 \mu \mathrm{Hz}$, where the error is the FWHM of the peak. 


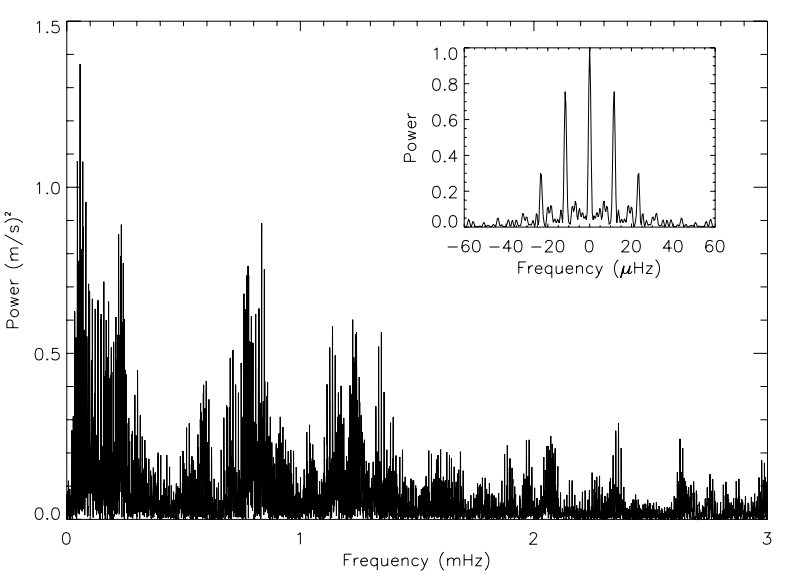

Fig. 2. The power spectrum of the weighted data. A power excess around $1 \mathrm{mHz}$ is evident. The inset shows the power spectrum of the window function for a sine-wave signal of amplitude $1 \mathrm{~m} \mathrm{~s}^{-1}$, sampled in the same way as the observations. The power units are the same as those of the main figure.

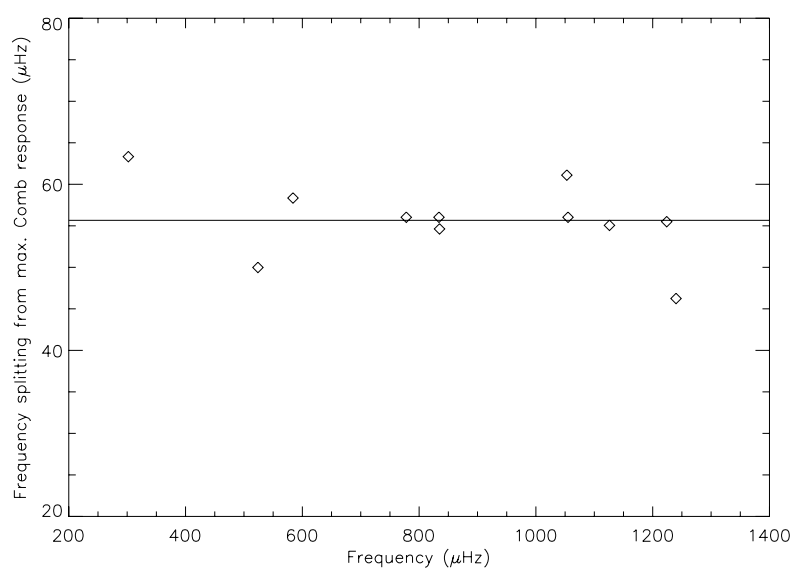

Fig. 3. The first-order spacing calculated over several frequency ranges, as determined from the comb-response analysis. The line shows the mean value of $55.7 \mu \mathrm{Hz}$, with a standard error of $\pm 1.4 \mu \mathrm{Hz}$.

\subsection{Oscillation frequencies and mode amplitude}

By assuming the $56 \mu \mathrm{Hz}$ regularity as genuine, we attempted to identify the oscillation frequencies directly from the PS. We used the following procedure to extract the frequencies ("standard" extraction): i) we found the largest peak, in the frequency range $0.5-1.5 \mathrm{mHz}$, then subtracted the corresponding sine-wave function from the time-series, and finally recomputed the PS; ii) we repeated this procedure for the new largest peak in the same range of frequency, the procedure being stopped when there were no peaks with amplitude larger than $0.29 \mathrm{~m}^{2} \mathrm{~s}^{-2}$, namely larger than $3 \sigma$, where $\sigma$ is the white noise evaluated in the PS between $2.5-3 \mathrm{mHz}$. The extracted frequencies are plotted in the echelle diagram shown in Fig. 5. Owing to single site observations, we need to take the daily alias of $11.57 \mu \mathrm{Hz}$ into account (see the spectral window in Fig. 2) in order to identify the modes in the echelle diagram; in fact, we cannot know a priori whether the frequencies, selected by the iterative procedure described above, are the correct ones or just aliases. Therefore, instead of shifting the frequencies by the daily alias, which might lead to an arbitrary identification of the modes for each shift,

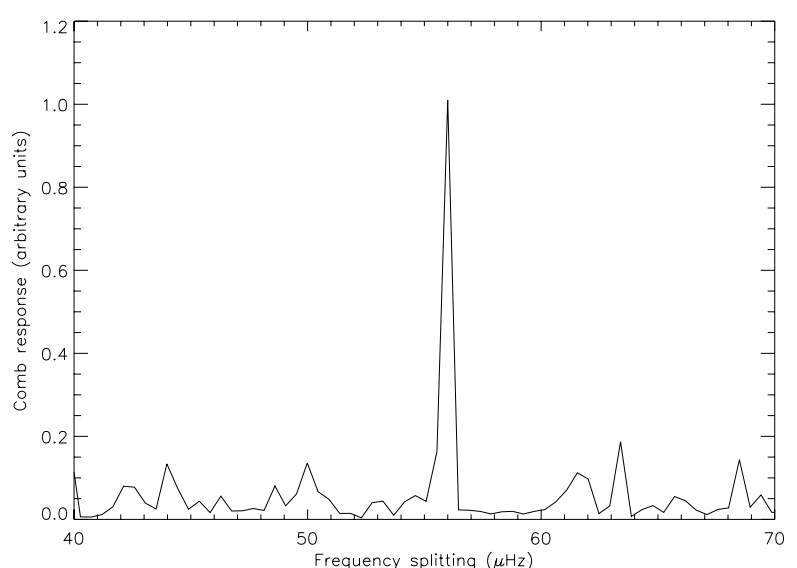

Fig. 4. The cumulative comb-response obtained as the sum of the individual comb-responses for each central frequency $v_{0}$. The peak is centered at $\Delta v=56 \pm 1 \mu \mathrm{Hz}$. The error is the FWHM of the peak.

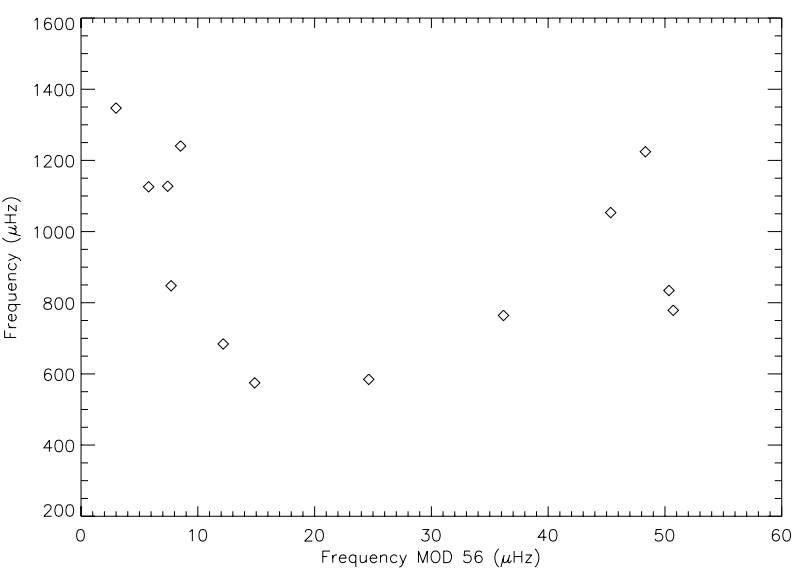

Fig. 5. The echelle diagram as derived from the "standard" extraction of frequencies.

we prefer to use a procedure ("modified" extraction) that relies on two assumptions only: i) the largest peak in the range of interest is a true pulsation mode; ii) the large-separation value is $56 \mu \mathrm{Hz}$. We operated in the same way as in the point i) of the "standard" extraction, then defined a new searching spectral region centered at a distance of $56 \mu \mathrm{Hz}$ from the first component, the extension of which was $2 \mu \mathrm{Hz}$, a suitable frequency span related to spectral resolution. When this region contained a peak with an amplitude larger than $3 \sigma$, we identified this peak as the second component to be cleaned and recomputed the PS. This process was repeated, shifting frequency back and forth by multiples of $56 \mu \mathrm{Hz}$; and once the whole region of interest had been covered, the remaining peak was identified and the process restarted with a new set of searching regions separated again by $56 \mu \mathrm{Hz}$, the procedure being stopped when all remaining peaks were below the $3 \sigma$ threshold. This is the same procedure as used by Kjeldsen et al. (1995) for $\eta$ Boo, which allows the mode frequencies to be determined except for the daily alias that could cause a shift of $\pm 11.57 \mu \mathrm{Hz}$. The frequencies extracted with this procedure are shown in Table 1 and the related echelle diagram in Fig. 6. The large and small frequency separations, and the constant $\varepsilon$ have been determined by means of a least-square best fit of frequencies with the asymptotic relationship (1), obtaining: $\Delta v=55.90 \pm 0.08 \mu \mathrm{Hz}, \delta v_{02}=7.1 \pm 1.3 \mu \mathrm{Hz}, \varepsilon=1.913 \pm 0.025$, 
Table 1. Prominent peaks in the power spectrum of Procyon identified as mode oscillation frequencies (in $\mu \mathrm{Hz}$ ) in terms of $n$ and $l$.

\begin{tabular}{cccc}
\hline \hline$n^{\dagger}$ & $l=0$ & $l=1$ & $l=2$ \\
\hline 7 & & 524.5 & \\
9 & 608.4 & & \\
11 & & & 770.0 \\
12 & 778.7 & $(808.6)^{\ddagger}$ & \\
13 & 834.3 & & \\
18 & 1114.2 & 1138.8 & \\
20 & 1224.2 & 1252.0 & \\
22 & 1335.4 & & \\
\hline
\end{tabular}

$\dagger$ Deduced from the fit to the asymptotic relationship (1).

$\ddagger$ Excluded from the fit because of its large deviation from the asymptotic relationship, though the related peak in the power spectrum is a high $S / N$ one.

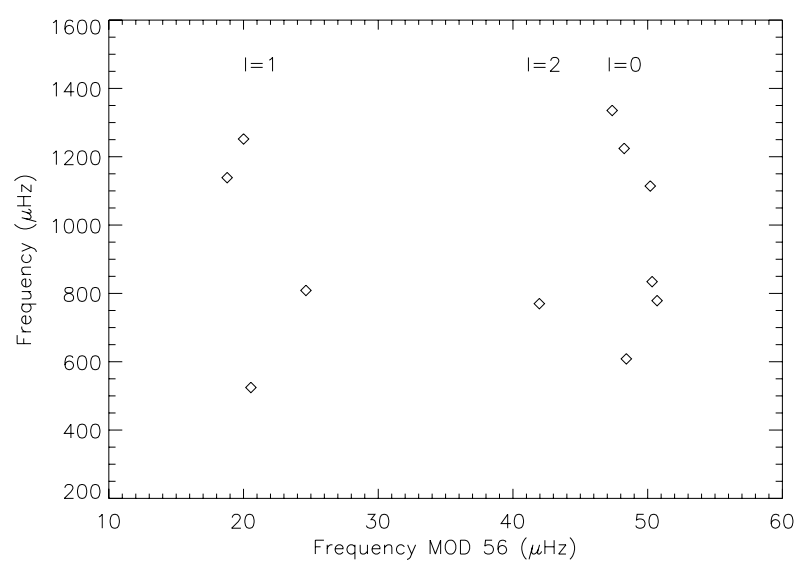

Fig. 6. The echelle diagram as derived from the "modified" extraction of frequencies.

with an rms scatter per mode of $1.23 \mu \mathrm{Hz}$. In Table 1 we also give a possible mode with $l=1$ and $n=12$ at $808.6 \mu \mathrm{Hz}$; however, this mode was excluded from the calculation of the fit because of its large deviation from the asymptotic relation, although the related peak in the power spectrum is a high $S / N$ one. Once $\Delta v$ has been determined, it is possible to construct a folded spectrum where the power in the PS is folded at the large separation, by using the frequency modulo of $55.90 \mu \mathrm{Hz}$. The result is shown in Fig. 7 where the mean power as a function of frequency modulo is plotted, and the positions of modes with $l=0,1,2$ are given, together with the positions of the daily side-bands, from which the detection of a $p$-mode structure in Procyon is evident.

The amplitude of individual modes can be estimated from the power concentrated in the PS, on assuming that only $l=0,1,2,3$ modes are detected, owing to the full disc observations. It is then possible to estimate the amplitude per mode necessary for producing the observed power level. Following the procedure described in Kjeldsen et al. (2005), we found a mean amplitude of the $p$-mode peak power, for the modes with $l=0,1$ in the frequency interval $0.6-1.4 \mathrm{mHz}$, of $0.45 \pm 0.07 \mathrm{~m} \mathrm{~s}^{-1}$, which is a value twice higher than the solar one and one in very good agreement with the amplitudes estimated by Brown et al. (1991). Our velocity amplitude can be transformed in intensity amplitude by using the Kjeldsen \& Bedding (1995) method, and obtaining the value of $7.3 \pm 1.1 \mathrm{ppm}$ per mode $(l=0,1)$ at $5500 \AA$, in good agreement with the WIRE (Bruntt et al. 2005) and upper-limit MOST (Matthews et al. 2004) measurements. The results are summarized in Fig. 8 where the velocity amplitude per mode

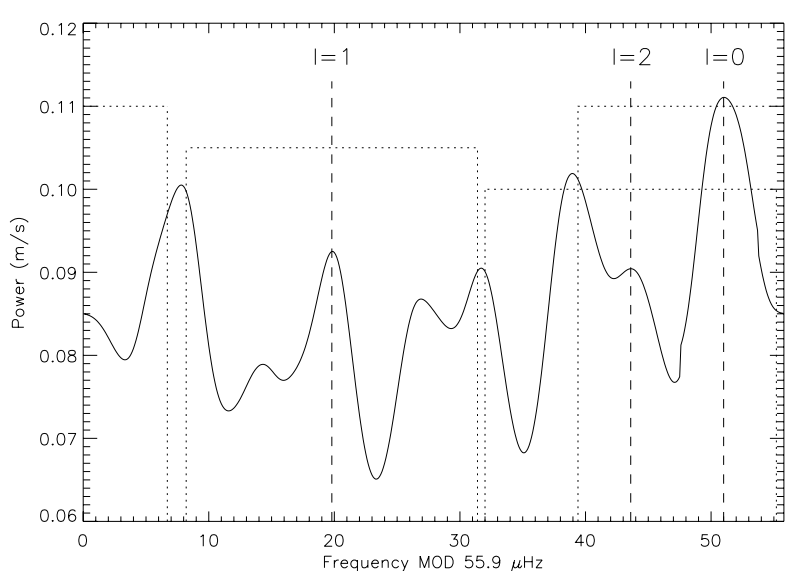

Fig. 7. The mean power folded at the large-separation frequency as a function of frequency modulo of $55.90 \mu \mathrm{Hz}$. The positions of the modes with $l=0,1,2$ are indicated, together with the positions of the daily side-bands.

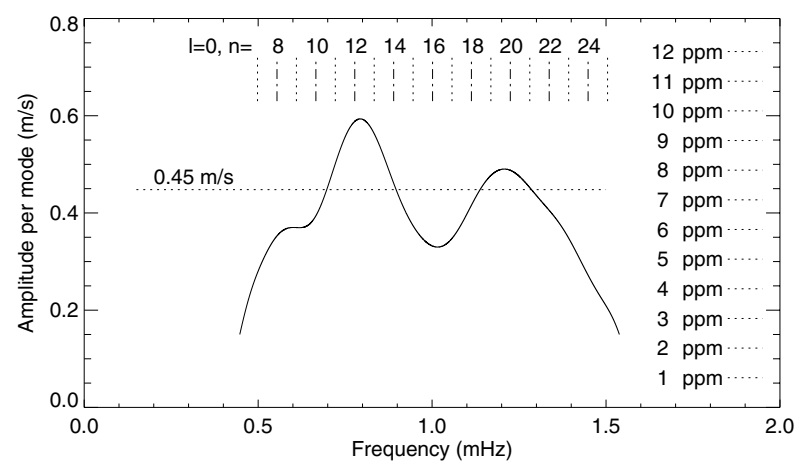

Fig. 8. Velocity amplitude per mode $(l=0,1)$ as a function of frequency. The horizontal line at $0.45 \mathrm{~m} \mathrm{~s}^{-1}$ represents the mean amplitude of the $p$-mode peak power in the frequency interval $0.6-1.4 \mathrm{mHz}$. The scale of intensity is also given, together with the positions of $l=0$ modes, as derived from asymptotic relationship (1).

is shown as a function of frequency, together with the intensity scale and the equispaced positions of $l=0$ modes, as derived from the relationship (1) into which the determined values of $\Delta v$ and $\varepsilon$ were inserted. In Fig. 8 , a dip at $1 \mathrm{mHz}$ is clearly visible, which confirms a previously reported result of Bedding \& Kjeldsen (2006) and whose presence is probably related to the damping rate of the modes (Houdek et al. 1999).

\subsection{Comparison with other frequency determinations}

Our results are directly comparable to those of Eggenberger et al. (2004), as given in their Table 3, where the identified frequencies are tabulated. We may also make a comparison with the raw extracted frequencies given by Eggenberger et al. (2004) in their Table 2. We are not able to make a direct comparison with the results by Martić et al. (2004), since they only provide a list of identified mode frequencies that depend on the fitting to theoretical models.

In order to perform the comparison with Eggenberger et al. (2004), we applied the same least-square best fit as applied to our frequency data to those of Eggenberger et al. (2004) and derived the parameters of the asymptotic relationship which are: $\delta v_{02}=4.9 \mu \mathrm{Hz}, \varepsilon=1.537$. In this procedure the value of 
Table 2. Comparison between the Eggenberger et al. (2004) extracted raw frequencies (in $\mu \mathrm{Hz}$ ) and the asymptotic relation from the present study $\left(\Delta v=55.90 \mu \mathrm{Hz}, \delta v_{02}=7.1 \mu \mathrm{Hz}, \varepsilon=1.913\right)$.

\begin{tabular}{|c|c|c|c|c|c|}
\hline $\begin{array}{c}\text { Raw frq. } \\
v\end{array}$ & Correction & $\begin{array}{r}\text { Fit } \\
v\end{array}$ & $l$ & $n$ & This study \\
\hline 630.8 & & & & & Noise? \\
\hline 651.5 & $+11.6=663.1$ & 665.9 & 0 & 10 & \\
\hline 662.7 & & 665.9 & 0 & 10 & \\
\hline 683.5 & $+11.6=695.1$ & 691.5 & 1 & 10 & $?$ \\
\hline 720.6 & & 721.8 & 0 & 11 & \\
\hline 791.8 & $-11.6=780.2$ & 777.7 & 0 & 12 & 778.7 \\
\hline 797.9 & (809.5?) & 803.3 & 1 & 12 & $808.6 ?$ \\
\hline 799.7 & (811.3?) & 803.3 & 1 & 12 & $808.6 ?$ \\
\hline 828.5 & & 826.5 & 2 & 12 & \\
\hline 835.4 & & 833.6 & 0 & 13 & 834.3 \\
\hline 856.2 & & 859.2 & 1 & 13 & \\
\hline 859.8 & & 859.2 & 1 & 13 & \\
\hline 911.4 & & 915.1 & 1 & 14 & ? \\
\hline 929.2 & $+11.6=940.8$ & 938.3 & 2 & 14 & \\
\hline 1009.7 & $-11.6=998.1$ & 1001.3 & 0 & 16 & \\
\hline 1027.1 & & 1026.9 & 1 & 16 & \\
\hline 1123.3 & $-11.6=1111.7$ & 1113.1 & 0 & 18 & 1114.2 \\
\hline 1131.1 & & & & & Noise? \\
\hline 1137.0 & & 1138.7 & 1 & 18 & 1138.8 \\
\hline 1186.0 & $+11.6=1197.5$ & 1194.6 & 1 & 19 & \\
\hline 1192.4 & & 1194.6 & 1 & 19 & \\
\hline 1234.8 & $-11.6=1223.2$ & 1124.9 & 0 & 20 & 1224.2 \\
\hline 1251.8 & & 1250.5 & 1 & 20 & 1252.0 \\
\hline 1265.6 & $+11.6=1277.2$ & 1273.7 & 2 & 20 & \\
\hline 1337.2 & & 1336.7 & 0 & 22 & 1335.4 \\
\hline 1439.0 & & 1141.4 & 2 & 23 & \\
\hline 1559.5 & & 1560.3 & 0 & 26 & \\
\hline
\end{tabular}

$\Delta v$ was taken fixed to the value given by Eggenberger et al. (2004), $\Delta v=55.5 \mu \mathrm{Hz}$. If one calculates the frequencies corresponding to the two asymptotic solutions, the one in the present paper and the one we obtained by fitting to the Eggenberger et al. (2004) data, we find that most of the calculated frequencies, for $l=0,1$, agree within $3 \mu \mathrm{Hz}$. We find that $l=0$, $n=9-24$ modes from the Eggenberger et al. (2004) asymptotic relation correspond to $l=1, n=8-23$ modes from the asymptotic relation in the present study. We also find that $l=1$, $n=7-22$ (Eggenberger et al. 2004) modes correspond to $l=0$, $n=7-22$ modes identified by us. We conclude that we and Eggenberger et al. (2004) are detecting signals from the same underlying p-modes. If we look at all the raw extracted frequencies given in Table 2 of Eggenberger et al. (2004), it appears striking that most of those frequencies may be identified by using the asymptotic solution deduced from the present study. In Table 2 we compare the Eggenberger et al. (2004) raw frequencies with the asymptotic relation from the present study $(\Delta v=55.90 \mu \mathrm{Hz}$, $\delta v_{02}=7.1 \mu \mathrm{Hz}, \varepsilon=1.913$ ).

It is interesting to note that 8 out of 11 frequencies determined by us match, within a few $\mu \mathrm{Hz}$, those listed in the Table 2 of Eggenberger et al. (2004) well, but with a different mode identification. Therefore we detected signals from the same frequencies as Eggenberger et al. (2004), but extracted the $p$-mode structure in a slightly different way, reaching two different solutions, and therefore identifications, for the extracted frequencies.

If we use our solution, $\Delta v=55.90 \mu \mathrm{Hz}, \delta v_{02}=7.1 \mu \mathrm{Hz}$, $\varepsilon=1.913$, to verify to what extent it matches the Eggenberger et al. (2004) frequencies, we find that 16 frequencies match our solution, 5 need a shift of $+11.6 \mu \mathrm{Hz}, 4$ need a shift of $-11.6 \mu \mathrm{Hz}$, and only 2 do not match the solution. Otherwise, if we use the Eggenberger et al. (2004) solution, $\Delta v=55.50 \mu \mathrm{Hz}$, $\delta v_{02}=4.9 \mu \mathrm{Hz}, \varepsilon=1.537$, we find that 12 frequencies match this solution, 8 need a shift of $+11.6 \mu \mathrm{Hz}, 3$ need a shift of $-11.6 \mu \mathrm{Hz}$, and 4 do not match the solution. This means that the frequencies detected by Eggenberger et al. (2004) provide a slightly better fit to our solution than to their solution.

This statement is supported by the fact that, if we only look at those 12 frequencies that fit, without shift, the Eggenberger et al. (2004) asymptotic relationship and compare them to the Eggenberger et al. (2004) 16 raw frequencies that fit the solution found in the present study, we see that the scatter for the 16 frequencies is about $10 \%$ lower when we use our asymptotic relation. Moreover, Eggenberger et al. (2004) identify the 50\% of those 12 frequencies that fit its relation as $l=2$ modes, while the $l=1$ modes should instead show the highest amplitude. However, since the two solutions provide basically the same frequencies, one should of course be cautious before claiming that one solution is far better than the other one.

\subsection{Mode lifetimes}

Solar-like $p$-mode oscillations in Procyon are presumably excited stochastically by the action of the surface convection (Houdek et al. 1999) as in the case of the Sun's oscillations. Therefore it is interesting to estimate the lifetime of modes for verifying the excitation mechanism that in turn determines their observed spectral width and amplitude. A coherent, over-stable, long-lived pulsation mode would concentrate all its power in a small frequency bin, while a stochastically, short-lived excited mode would spread its power over a larger frequency interval. In the case of the Sun, the mode lifetime is about $3 \pm 1 \mathrm{~d}$ at the peak amplitude of $3 \mathrm{mHz}$, as estimated by fitting a model to the power distribution for each mode in the PS as in Chaplin et al. (1997). Their technique requires observations for a significantly longer time than the mode lifetime, which is not necessarily consistent with our observing period of 6 nights.

The method we use in the present analysis is based on the frequency scatter per identified mode (Bedding et al. 2004; Kjeldsen et al. 2005), as calculated in Sect. 3.2. We ran 8400 individual simulations to determine the rms scatter of the detected frequencies as a function of the mode lifetime, by using the De Ridder et al. (2006) simulator and neglecting rotational splittings both because Procyon is a slow rotator and the majority of modes used are radial. The simulations contain the correct amplitude of modes, noise level, and number of detected modes out of the total number of modes contained in the $p$-mode spectrum, as determined from the asymptotic relationship (1) in the observed frequency interval. The fit represents a model that contains two components: the frequency spread, which is essentially the width of the Lorentzian profile, and the scatter of coherent oscillations, which corresponds to an infinite mode lifetime. The results are reported in Fig. 9 where the rms scatter of the 10 considered frequencies, out of the 11 identified (see Sect. 3.2), is plotted vs. mode lifetime. Since the mode frequency scatter determined in Sect. 3.2 turns out to be $1.23 \mu \mathrm{Hz}$, it is easily seen from Fig. 9 that the corresponding lifetime is $2.0 \pm 0.4 \mathrm{~d}$. The observed frequency scatter in Procyon is noticeably larger than is consistent with coherent oscillations, ruling out this possibility, and the related mode lifetimes appear to be slightly shorter than those observed in the Sun, where the oscillations are excited by the surface turbulent convection. The present data strongly indicate that oscillations in Procyon are not coherent, but damped and re-excited by a mechanism similar to what is operating in the Sun, as expected. 


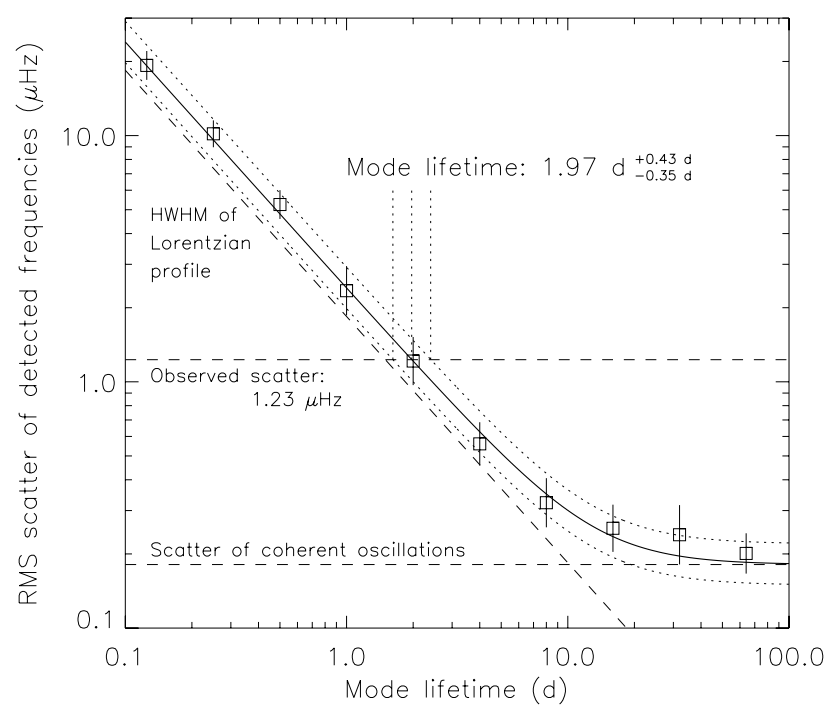

Fig. 9. The rms scatter of the 10 identified frequencies as a function of mode lifetime obtained by several thousand simulations. The intersection of the horizontal line of the observed scatter with the solid curve obtained by simulations indicates that the mode lifetime is $1.97_{-0.35}^{+0.43} \mathrm{~d}$. The horizontal line of coherent oscillation scatter, corresponding to an infinite mode lifetime, is also indicated. The frequency spread reflects essentially the width of the Lorentzian profile.

The results of the previous method for determining the mode lifetimes are corroborated by a different analysis we performed based on the amplitude of the highest peaks in the PS, with the idea that the amplitude of modes are also affected by their lifetimes, in the sense that, when the peak amplitude is smaller, the mode lifetime is shorter, while when it is larger the mode lifetime is longer. However, since there is not a simple relationship between amplitude and lifetime, we used a data simulation based on several thousand runs that allows a direct comparison between the observed properties of the time-series and the properties of the oscillation modes in order to establish a calibrated relationship between the peak height and the mode lifetime (De Ridder et al. 2006). Compared to the previous one, this method has the advantage of being independent of mode identification but the disadvantage of being less accurate. The result is reported in Fig. 10, where we show the amplitude, with $1 \sigma$ error bars, of the 6 largest-amplitude detected modes with $l=0,1,2$ as a function of the mode lifetime. In Fig. 10 the intersection of the horizontal line corresponding to that of the observed mode of largest amplitude (see Fig. 2) with the simulated data gives an average mode lifetime $\simeq 2 \mathrm{~d}$, in good agreement with the frequency scatter method but with a much larger uncertainty. It is interesting to note the expected trend of mode amplitudes as a function of their lifetimes.

The effects of frequency scatter and mode amplitude on mode lifetime is evident in Figs. 11 and 12, where simulations concerning $10 \mathrm{~d}$ continuous observation campaigns are reported for mode lifetimes of $2 \mathrm{~d}$ and $0.5 \mathrm{~d}$, respectively. In these two figures the upper panel shows the complete PS, while the lower one an enlarged portion of it from $0.8 \mathrm{mHz}$ to $1.0 \mathrm{mHz}$. From our simulations it is clear that the frequencies of long-lived modes are much better defined, or less scattered, and have larger amplitudes than those of short-lived modes.

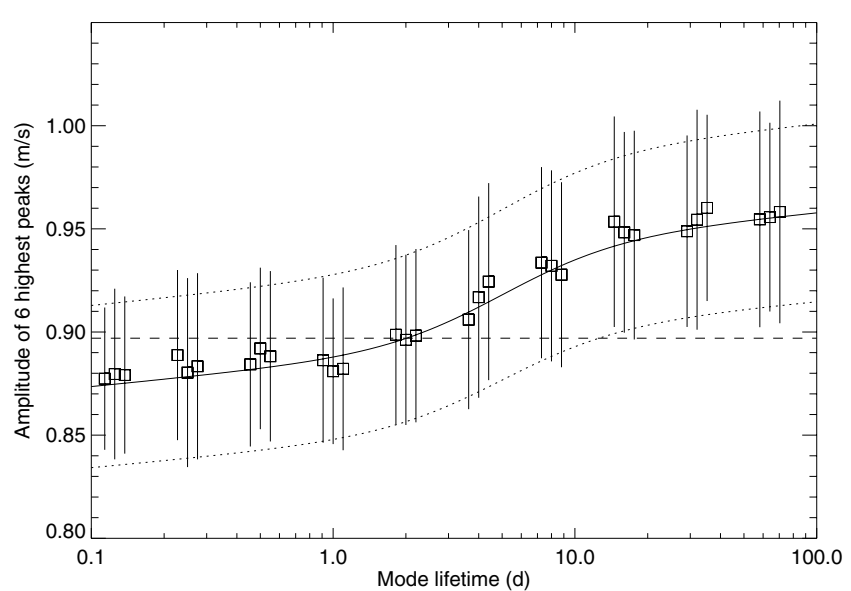

Fig. 10. The amplitude of the 6 largest amplitude detected modes, for $l=0,1,2$ as a function of the mode lifetime. The bars indicate the $1 \sigma$ error.
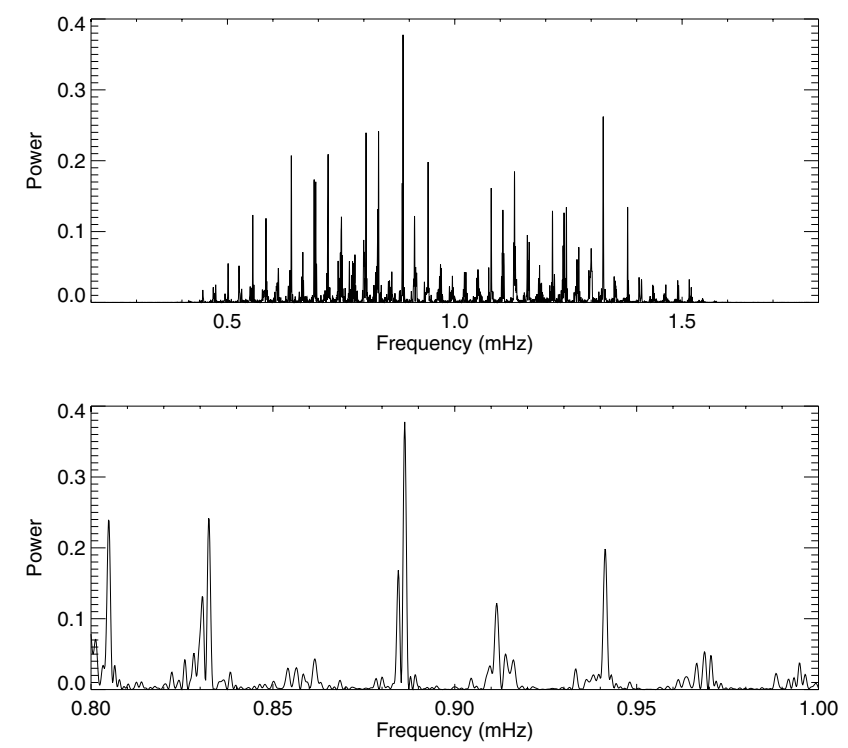

Fig. 11. Simulated Procyon $p$-mode power spectrum obtained by using the De Ridder et al. (2006) simulator, not including noise and rotational splittings. The length of the time-series is $10 \mathrm{~d}$ and the mode lifetime is $2 \mathrm{~d}$.

\section{Line equivalent width measurements and analysis}

As already mentioned, the red part of the echelle spectrum of Procyon is insensitive to the iodine cell calibration and therefore the search for stellar pulsations can be performed only by measuring $E W$. The presence of telluric lines and limited number of strong lines sensitive to temperature restricted our analysis to the spectral lines reported in Table 3 (e.g. $\mathrm{H}_{\alpha}$ is located at the border of spectral orders and is therefore useless for accurate $E W$ measurements).

\subsection{Power spectrum}

Since the expected variations in the $E W$ induced by the stellar pulsations are on the order of a few ppm, we adopted an accurate approach for the measurement of the $E W$, following 

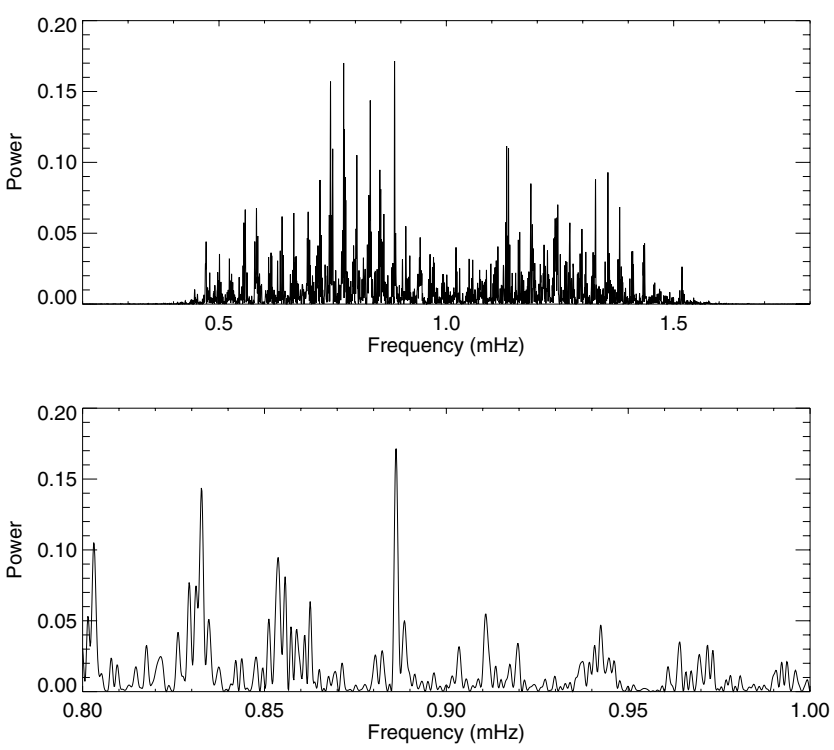

Fig. 12. The same simulation as in Fig. 11, but for a mode lifetime of $0.5 \mathrm{~d}$. Note that the frequency spreads are larger and peak amplitudes smaller than those of the modes seen in Fig. 11.

Table 3. Spectral line wavelengths used for $E W$ measurements.

\begin{tabular}{cc}
\hline \hline Wavelength $(\AA)$ & Spectral lines \\
\hline 6393.4 & $\mathrm{Fe} \mathrm{I}$ \\
6436.7 & $\mathrm{Ca} \mathrm{I}$ \\
6462.4 & $\mathrm{Ca} \mathrm{I} / \mathrm{Fe} \mathrm{I}$ \\
6546.0 & $\mathrm{Fe} \mathrm{I}$ \\
6574.9 & $\mathrm{Fe} \mathrm{I}$ \\
6592.7 & $\mathrm{Fe} \mathrm{I}$ \\
6643.4 & $\mathrm{Ni} \mathrm{I}$ \\
6677.8 & $\mathrm{Fe} \mathrm{I}$ \\
6717.5 & $\mathrm{Ca} \mathrm{I}$ \\
\hline
\end{tabular}

Kjeldsen et al. (1995). Instead of fitting the line profile for determining the $E W$, we computed the flux in three artificial filters, analogously to Strömgren $\mathrm{H}_{\beta}$ photometry. The first filter was centered on the considered line at the wavelength $\lambda_{0}$, with a band-width $\Delta \lambda_{0}$, and the other two on the near continuum with respect to the line at $\lambda_{0}$, one placed on the red region at $\lambda_{\mathrm{r}}$ with band-width $\Delta \lambda_{\mathrm{r}}$ and the other on the blue one at $\lambda_{\mathrm{b}}$, with a bandwidth $\Delta \lambda_{\mathrm{b}}$. We then computed the $E W$, or more precisely the line-index, in terms of the radiation flux measured in the selected filters $\mathcal{F}(\Delta \lambda)$ :

$E W=\left[\frac{\mathcal{F}\left(\Delta \lambda_{0}\right)}{\Delta \lambda_{0}}\right] \cdot\left[\frac{\Delta \lambda_{\mathrm{r}}+\Delta \lambda_{\mathrm{b}}}{\mathcal{F}\left(\Delta \lambda_{\mathrm{r}}\right)+\mathcal{F}\left(\Delta \lambda_{\mathrm{b}}\right)}\right]$

for two different central filter widths, $\Delta \lambda_{0}^{1}$ and $\Delta \lambda_{0}^{2}$, in order to obtain two values for the $E W, E W_{1}$, and $E W_{2}$, from which the quantity $E W_{\text {best }}=E W_{1}^{m+1} / E W_{2}^{m}$ can be constructed. The aim of this procedure is to construct a quantity that is less sensitive to the continuum fluctuations, determining $m$ so as to minimize the scatter of the measurements in a similar way as for the RV analysis. The procedure was repeated for all the lines reported in Table 3, and then, in order to improve the $S / N$, we combined all the $E W_{\text {best }}$ 's obtained for the examined lines, assuming the same sensitivity to temperature changes for them, and derived a weighted mean value. In Figs. 13 and 14 we show the time-series

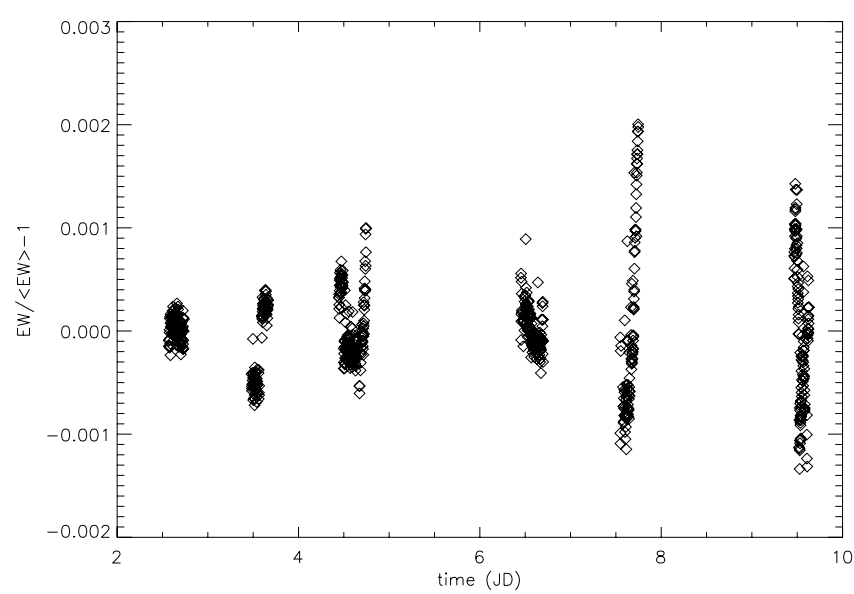

Fig. 13. Time series of $E W$ measurements as derived from the combination of all the lines reported in Table 3.

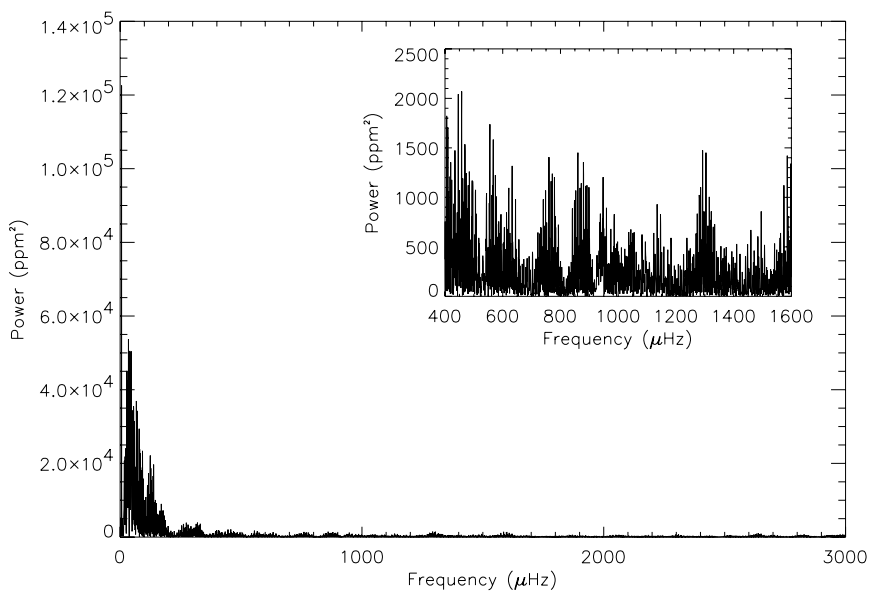

Fig. 14. The weighted power spectrum $E W_{\text {best }}-\left\langle E W_{\text {best }}\right\rangle$ derived from the combination of the measurements of all the lines of Table 3 . There is no power excess in the range $0.5-1.5 \mathrm{mHz}$, but the background power in the range $0.2-0.7 \mathrm{mHz}$ could be a signature of stellar granulation.

and the weighted PS of the $E W_{\text {best }}$, respectively. The weights adopted are $w_{i}=1 / \sigma_{i}^{2}$, where the $\sigma_{i}$ 's are the standard deviations of the data.

\subsection{Granulation noise}

The combined power spectra do not show evidence of power excess in the range $0.5-1.5 \mathrm{mHz}$, as is clear from Fig. 14. However, at low frequencies, some concentration of power is apparent, similar to the background power reported by Kjeldsen et al. (1999) for $\alpha$ Cen A and attributed to stellar granulation. Analogously to Kjeldsen et al. (1999), here we attempt to estimate the amount of power related to Procyon granulation. In this procedure we optimize the $S / N$ in the PS by computing the weighted means of the measurements, by using weights that minimize the mean noise for each line in the interval $0.2-0.7 \mathrm{mHz}$, and then by rescaling the weights for each line in such a way that the weights, $w_{i}$, satisfy the relationship $\sum w_{i}=$ $1 / \mathcal{A}$, where $\mathcal{A}$ is the mean of the peak amplitudes in the range $0.2-0.7 \mathrm{mHz}$. 


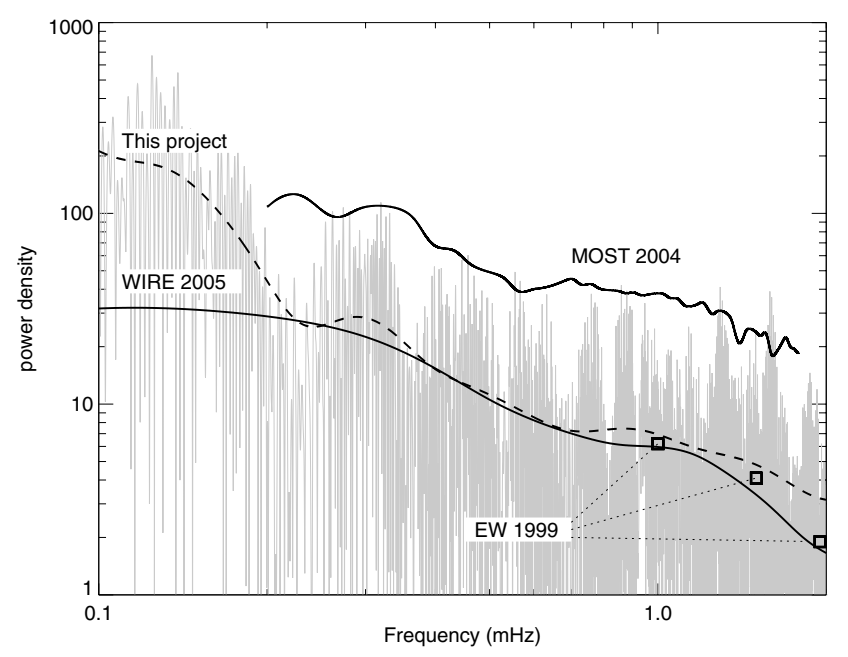

Fig. 15. Smoothed power density spectra (PDS) of Procyon granulation noise as obtained from SARG (dashed line, this project) and AAT/ESO $E W$ 1999, squares, (Kjeldsen et al. 1999) spectroscopy, and MOST (Matthews et al. 2004) and WIRE (Bruntt et al. 2005) space photometry (continuous lines). The PDS derived from the PS of Fig. 14 is shown in the background. Power density is expressed in $\mathrm{ppm}^{2} \mu \mathrm{Hz}^{-1}$.

To evaluate the power generated by the granulation of the star in the PS of the $E W$ 's and to make a comparison with data obtained with different methods we need to define a quantity independent of the temporal length of the data, which is obtained by computing the power density spectrum (PDS), a measure of the power per frequency resolution element that is therefore independent of the length and sampling of the time-series:

$\operatorname{PDS}(v)=A^{2}(v)\left[\int_{0}^{v_{\mathrm{N}}} W^{2}(v) \mathrm{d} v\right]^{-1}$

where $A(v)$ is the amplitude (in ppm) in the PS, $W(v)$ the window function, and $v_{\mathrm{N}}$ the Nyquist frequency. The result of our analysis is shown in Fig. 15, the smoothed power density spectrum of Procyon granulation noise (this project), and the PDS of the PS shown in Fig. 14.

\subsection{Comparison with other granulation noise determinations}

The literature reports three previous claims of granulation noisepower detection in Procyon. The first is by Kjeldsen et al. (1999), who found a granulation noise slightly above the solar level in the frequency interval $1-2 \mathrm{mHz}$, by using the Balmer $E W$ 's (Fig. 15; EW 1999). The other two claims concern space photometric measurements, instead of spectroscopic, carried out by the satellites MOST (Matthews et al. 2004), operating in the bandwidth 3700-7000 , and WIRE (Bruntt et al. 2005), operating with the white light star tracker.

While our measurements are directly comparable with the spectroscopic ones of Kjeldsen et al. (1999), in order to compare them with the photometric ones, we need to pass from intensities $I$ to temperatures $T$ and from there to $E W$ 's. This is accomplished by using appropriate sensitivity indices; for intensity, we used $\partial \ln I / \partial \ln T=4.5$ (Kjeldsen \& Bedding 1995), and $\partial \ln (E W) / \partial \ln T=-7.8$ for $E W$, as deduced from the analysis of Bedding et al. (1996) for Fe I lines at $T=6500 \mathrm{~K}$. The results are shown in Fig. 15, where MOST and WIRE PDF curves are plotted together with Kjeldsen et al. (1999) data and our curve.
Table 4. Properties of the $p$-mode oscillations in Procyon.

\begin{tabular}{lc}
\hline \hline Large separation $\Delta v$ & $55.90 \pm 0.08 \mu \mathrm{Hz}$ \\
Small separation $\delta v_{02}$ & $7.1 \pm 1.3 \mu \mathrm{Hz}$ \\
Asymptotic relationship $\varepsilon$ & $1.913 \pm 0.025$ \\
Frequency scatter per mode & $1.23 \mu \mathrm{Hz}$ \\
$v(l=0, n=16)$ & $1001.3 \pm 0.4 \mu \mathrm{Hz}$ \\
Mean amplitude per mode $(l=0,1)$ & $0.45 \pm 0.07 \mathrm{~m} \mathrm{~s}^{-1}$ \\
Mode lifetime & $2.0 \pm 0.4 \mathrm{~d}$ \\
\hline
\end{tabular}

It appears that, while WIRE and our distribution of power are mutually consistent, at least in the frequency interval $0.2-2 \mathrm{mHz}$, and corroborated by the few data of Kjeldsen et al. (1999), MOST shows a power density level higher by a factor of four or more. Uncertainties in sensitivity index calibration of $I$ and $E W$ with respect to $T$ may lead to some discrepancies, but they are not enough large to explain such a significant disagreement between MOST and other data. In order to render the MOST data comparable with ours, it should be $|\partial \ln (E W) / \partial \ln T|<4.5$ and $\partial \ln I / \partial \ln T>4.5$. This is unlikely because the value of $\partial \ln (E W)) / \partial \ln T$ adopted here has been derived by observations of the same lines we use here at the same temperature of Procyon (Bedding et al. 1996) and a value of $\partial \ln I / \partial \ln T>4.5$ disagrees with the models of Bedding et al. (1996). This seems to imply that MOST did not detect granulation noise, probably because of some instrumental or stellar spurious signal, as suggested by Bruntt et al. (2005).

In contrast to the first spectroscopic determinations of Kjeldsen et al. (1999) relative to a few points in the high frequency side of the granulation noise PDS, our $E W$ determinations constitute the first spectroscopic measurements of Procyon granulation in the frequency range $0.1-2.0 \mathrm{mHz}$, so providing an independent measure of and establishing an upper limit to granulation noise power. The fact that at low frequencies, below $0.2 \mathrm{mHz}$, our power level is higher than the one shown by WIRE might be caused partially because we did not correct the WIRE data at low frequencies for the effect of high-pass filter and partially because of the possible effect of stellar activity in Procyon that affects spectral lines but not the continuum.

\section{Conclusions}

The analysis of the PS of Procyon obtained by the SARG highresolution spectrograph unequivocally shows the presence of a solar-like $p$-mode spectrum, as clearly demonstrated in Fig. 7. The main seismic properties of Procyon are summarized in Table 4.

We identified 11 individual $p$-mode frequencies with $l=$ $0,1,2$ and $7 \leq n \leq 22$ with a good margin of certainty in the frequency range $500-1400 \mu \mathrm{Hz}$, as reported in Table 1, which are largely consistent with those found by Eggenberger et al. (2004).

The large frequency separation deduced from the fit of 10 , out of the 11 identified frequencies, to the asymptotic relationship (1) is consistent with the results from the comb response analysis, both individual and cumulative, and its value agrees fairly well with $\Delta v=55.5 \mu \mathrm{Hz}$ determined by Eggenberger et al. (2004); but it shows some discrepancy with what was determined by Martić et al. (2004), $\Delta v=53.6 \mu \mathrm{Hz}$, although in a previous article (Martić et al. 1999) the authors found $\Delta v=55.6 \mu \mathrm{Hz}$, as is evident from their Fig. 12 . 
The large frequency separation we have determined is consistent with an evolutionary model for Procyon, constructed with solar chemical abundance, elemental diffusion, and mass loss, having a mass of $1.48 M_{\odot}$, an age of $1.8 \mathrm{~Gy}$, a luminosity of $6.9 L_{\odot}$, a radius of $2.04 R_{\odot}$, and a surface convection zone that is $8 \%$ of the stellar radius (Bonanno et al. 2007). This is in very good agreement with other theoretical studies of Procyon A (Barban et al. 1999; Chaboyer et al. 1999; Eggenberger et al. 2005).

The small frequency separation we determined from one $l=2$ and three $l=1$ modes is larger by $1.6 \sigma$ than what was determined by Martić et al. (2004), $\delta v_{02}=5.1 \mu \mathrm{Hz}$, and what we derived from Eggenberger et al. (2004) data, $\delta v_{02}=4.9 \mu \mathrm{Hz}$, but our value is not strongly constrained by our measurements, as only a few modes with $l \neq 0$ were identified.

The mean amplitude per mode, for modes with $l=0,1$, is about 0.45 , twice larger than the solar one. The mode lifetime was determined by means of two independent methods whose results are mutually consistent and give a lifetime of about $2 \mathrm{~d}$. This short lifetime rules out the possibility of a coherent, overstable excitation of modes in Procyon, but it indicates that oscillations are excited stochastically by turbulent convection as in the case of the Sun.

We used the red part of the observed spectrum and the measurement of the $E W$ 's to determine an upper limit to granulation noise-power that turned out to agree with previous spectroscopic (Kjeldsen et al. 1999) and space photometric (Bruntt et al. 2005) measurements, but significantly contrasting with MOST data (Matthews et al. 2004).

Our RV data are available upon request from the first author of the present article.

Acknowledgements. This work was partially supported by the Italian Ministry of University and Scientific Research, under the contract PRIN 2004024993.

\section{References}

Allende Prieto, C., Asplund, M., García López, R. J., \& Lambert, D. 2002, ApJ, 567,544

Barban, C., Michel, E., Martić, M., et al. 1999, A\&A, 350, 617

Bedding, T. R., \& Kjeldsen, H. 2006, Mem. Soc. Astron. Ital., 77, 384

Bedding, T. R., Kjeldsen, H., Reetz, J., \& Barbuy, B. 1996, MNRAS, 280, 1155

Bedding, T. R., Kjeldsen, H., Butler, R. P., et al. 2004, ApJ, 614, 680

Bedding, T. R., Kjeldsen, H., Bouchy, F., et al. 2005, A\&A, 432, L43

Bonanno, A., Küker, M., \& Paternò, L. 2007, A\&A, 462, 1031

Bouchy, F., Maeder, A., Mayor, M., et al. 2004, Nature, 342, brief communications

Brown, T. M., Gilliland, R. L., Noyes, R. W., \& Ramsey, L. W. 1991, ApJ, 368, 599

Bruntt, H., Kjeldsen, H., Buzasi, D. L., \& Bedding, T. R. 2005, ApJ, 633, 440

Butler, R. P., Bedding, T. R., Kjeldsen, H., et al. 2004, ApJ, 600, L75

Chaboyer, B., Demarque, P., \& Guenther, D. B. 1999, ApJ, 525, L41

Chaplin, W. J., Elsworth, Y., Isaak, G. R., et al. 1997, MNRAS, 288, 623

Claudi, R. U., Bonanno, A., Leccia, S., et al. 2005, A\&A, 429, L17 (Paper I)

De Ridder, J., Arentoft, T., \& Kjeldsen, H. 2006, MNRAS, 365, 595

Eggenberger, P., Carrier, F., Bouchy, F., \& Blecha, A. 2004, A\&A, 422, 247

Eggenberger, P., Carrier, F., \& Bouchy, F. 2005, New Astron., 10, 195

Endl, M., Kürster, M., \& Els, S. 2000, A\&A, 353, L33

Gratton, R., Bonanno, G., Bruno, P., et al. 2001, Exp. Astron., 12, 107

Guenther, D. B., \& Demarque, P. 1993, ApJ, 405, 298

Houdek, G., Blamforth, N. J., Christensen-Dalsgaard, J., \& Gough, D. O. 1999, A\&A, 351, 382

Kjeldsen, H., \& Bedding, T. R. 1995, A\&A, 293, 87

Kjeldsen, H., Bedding, T. R., Viskum, M., \& Frandsen, S. 1995, AJ, 109, 1313

Kjeldsen, H., Bedding, T. R., Frandsen, S., \& Dall, T., 1999, MNRAS, 303, 579

Kjeldsen, H., Bedding, T. R., Butler, R. P., et al. 2005, ApJ, 635, 1281

Martić, M., Schmitt, J., Lebrun, J. C., Barban, C., et al. 1999, A\&A, 351, 993

Martić, M., Lebrun, J. C., Appourchaux, T., \& Korsennik, S. G. 2004, A\&A, 418, 295

Matthews, J. M., Kusching, R., Guenther, D. B., et al. 2004, Nature, 430, 51

Mosser, B., Maillard, J.-P., Mekarnia, D., \& Gay, J. 1998, A\&A, 340, 457

Régulo, C., \& Roca Cortés T. 2005, A\&A, 444, L5

Tassoul, M. 1980, ApJS, 43, 469 